\title{
Tetracycline Loaded Collagen/Hydroxyapatite Composite Materials for Biomedical Applications
}

\author{
Laura Cristina Rusu, ${ }^{1}$ Ioan Avram Nedelcu, ${ }^{2}$ Mădălina Georgiana Albu, ${ }^{3}$ \\ Maria Sonmez, ${ }^{3}$ Georgeta Voicu, ${ }^{2}$ Marius Radulescu, ${ }^{2}$ Denisa Ficai, ${ }^{2}$ Anton Ficai, ${ }^{2}$ \\ Meda-Lavinia Negrutiu, ${ }^{1}$ and Cosmin Sinescu ${ }^{1}$

\begin{abstract}
${ }^{1}$ Department of Technology of Materials and Devices in Dental Medicine, "Victor Babeş" University of Medicine and Pharmacy Timişoara, Piaţa Eftimie Murgu, No. 2, 300041 Timişoara, Romania
\end{abstract} \\ ${ }^{2}$ Faculty of Applied Chemistry and Material Science, Politehnica University of Bucharest, 1-7 Polizu Street, 011061 Bucharest, Romania \\ ${ }^{3}$ Collagen Department, Leather and Footwear Research Institute, 93 Ion Minulescu, 031215 Bucharest, Romania
}

Correspondence should be addressed to Mădălina Georgiana Albu; albu_mada@yahoo.com

Received 19 May 2015; Accepted 28 July 2015

Academic Editor: Akiko Obata

Copyright (C) 2015 Laura Cristina Rusu et al. This is an open access article distributed under the Creative Commons Attribution License, which permits unrestricted use, distribution, and reproduction in any medium, provided the original work is properly cited.

The paper describes the preparation, characterisation, and testing of tetracycline loaded collagen-carboxymethylcellulose/ hydroxyapatite ternary composite materials. The synthesis of this drug delivery system consists in two steps: the first step is the mineralization of collagen-carboxymethylcellulose gel while the second step corresponds to the loading of the ternary composite material with tetracycline. The obtained DDS is characterised by physicochemical, morphological, and release behaviour by using FTIR spectroscopy and microscopy, scanning electron microscopy, and UV-VIS spectroscopy. Based on the release study, it can be assumed that tetracycline is released in a prolonged way, assuring at least 6 days of antiseptic properties.

\section{Introduction}

Collagen (COLL) and hydroxyapatite (HA) are the main components of the bone [1]. This is why many materials designed for bone grafting and repair are based on collagen, hydroxyapatite, or composite materials containing the aforementioned components. The performances of these synthesized materials are strongly dependent on the composition and processing induced morphology [1-4].

Due to the compositional similitude with the bone, collagen/hydroxyapatite (COLL/HA) composite materials seem to be the most suitable material for bone engineering. Unfortunately, the lower properties of these materials comparing with natural bones made it necessary to improve the composition of these materials by adding third components [5] or to change the processing route in order to obtain certain morphology $[2,6,7]$.
The collagen-carboxymethylcellulose/hydroxyapatite (COLL-CMC/HA) was previously obtained in our group and seems to be proper material for bone grafting and repair [5]. The three components seem to be highly compatible, the FTIR maps obtained at wavelength corresponding to CMC (carboxymethylcellulose) $\left(715 \mathrm{~cm}^{-1}\right)$, COLL $\left(1655 \mathrm{~cm}^{-1}\right)$, and $\mathrm{HA}\left(1033 \mathrm{~cm}^{-1}\right)$ revealing a very homogeneous material. COLL-CMC/HA composite material containing both fibres (CMC) and particles (HA) as reinforcing agent is a true hybrid material, expecting superior mechanical properties compared to COLL/HA composite materials.

One of the most important shortcomings of the surgical intervention is related to the possibility of infections. This is why, in many surgical interventions, systemic administration of antibiotic is prescribed in both pre- and postoperative steps [8-10]. Unfortunately, systemic administration of antibiotics 
also involves systemic toxicity and high incidence of antibiotic resistance $[11,12]$. To counter the increasing antibiotic resistance there are two solutions meaning to produce new and new antibiotics or, much better, to use antibiotics in a rational way in order to avoid the development of antibiotic resistance. The most important ways to avoid antibiotic resistance development are to avoid the irrational use of antibiotics, to avoid the excessive use of antibiotics, to use local instead of systemic therapies, the rational choice of the used antibiotic, and so forth [13-17]. Tetracycline, being a broad-spectrum antibiotic indicated against many bacterial infections, was chosen to be used in our study.

The aim of this work is to reduce the amount of antibiotics (tetracycline) by a more rational way of use. For this purpose, tetracycline is embedded in the COLL-CMC/HA composite material, the final tetracycline loaded composite materials being able to assure the osteoregeneration and to assure the anti-infective properties without the need of systemic administration of antibiotic. The locoregional delivery of tetracycline is very important because the overall systemic side effects are limited or even removed.

\section{Materials and Methods}

2.1. Materials and Reagents. Type I fibrillar collagen gel with a molecular weight of about $300.000 \mathrm{Da}$ and acidic $\mathrm{pH}$ (2.6) was obtained in the Collagen Department of National Research \& Development Institute for Textiles and Leather as we previously described [18] and purified by dialysis, against water free of endotoxins.

Carboxymethylcellulose sodium salt (low viscosity) and tetracycline hydrochloride (BioReagent, suitable for cell culture) were purchased from Sigma-Aldrich (Saint Louis, USA) and were used without any further purification.

Hydroxyapatite was obtained in situ, in the presence of the carboxymethylcellulose-collagen gel. The precursors used for HA synthesis were calcium hydroxide (puriss. p.a.) and sodium phosphate monobasic monohydrate (ACS reagent), both purchased from Sigma-Aldrich (Steinheim, Germany).

\subsection{Synthesis of COLL/HA-Tetracycline Composite Material.} COLL/HA-tetracycline was obtained as schematically presented in Figure 1.

The first step is devoted to the preparation of the support material, the second step to the loading with tetracycline, and the last step to the drying. The support preparation consists in homogenisation of the collagen gel and carboxymethylcellulose (COLL:CMC weight ratio is $2: 1$ ) followed by mineralization with $\mathrm{Ca}(\mathrm{OH})_{2}$ and $\mathrm{NaH}_{2} \mathrm{PO}_{4} \cdot \mathrm{H}_{2} \mathrm{O}$ as previously presented $[5,7,19]$, cross-linked with glutaraldehyde $1 \%$ (reported to the dry collagen), and finally washing with distilled water (three times, each washing step taking $30 \mathrm{~min}$ ) to remove the soluble salts. The mineralization was performed in order to obtain COLL:CMC: HA composite support material with a mass ratio of $2: 1: 8$. The loading with tetracycline was done by absorbing the corresponding amount of tetracycline hydrochloride $(0.5 \mathrm{~g}$ tetracycline $/ 10 \mathrm{~g}$ composite) as aqueous solution. The drying can be done by

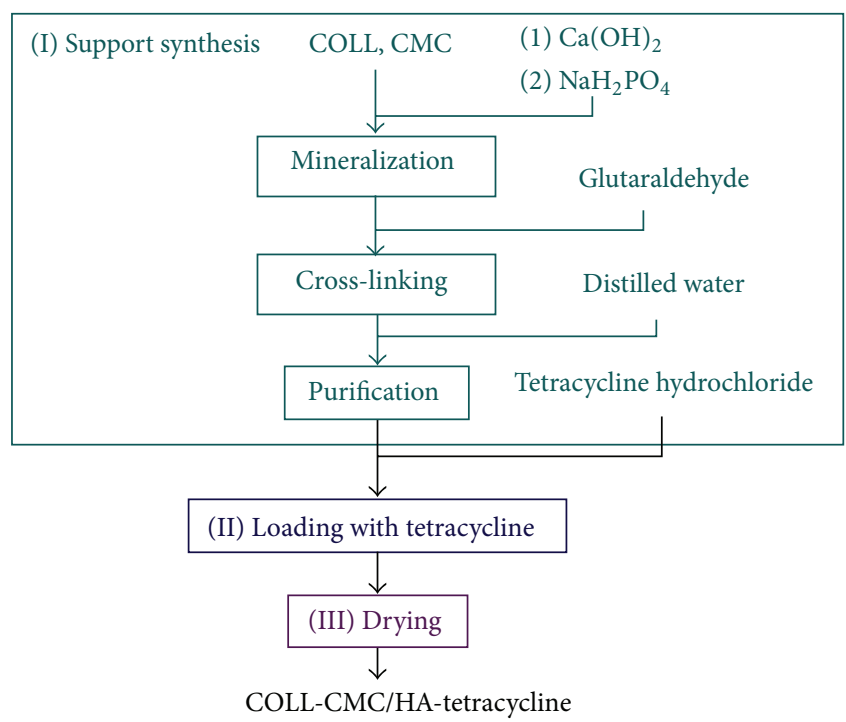

FIGURE 1: Schematic representation of preparation of COLLCMC/HA-tetracycline.

controlled air drying or by freeze-drying, depending on the desired properties, as previously described by Andronescu et al. [1].

2.3. Characterisation and Testing. After synthesis and freezedrying, the composite material was analyzed by using Fourier Transform-Infrared (FTIR) spectroscopy and microscopy and scanning electron microscopy (SEM). The release of tetracycline was monitored by using UV-VIS spectroscopy.

IR microscopy/spectroscopy was performed by using a Thermo FTIR Nicolet iN10 MX microscope; the spectra were recorded in ATR mode over the wave number range of $675-4000 \mathrm{~cm}^{-1}$, with a resolution of $4 \mathrm{~cm}^{-1}$. For a better identification of the peaks, the obtained spectra were resolved using a Gaussian-Lorentzian peak resolve procedure, with no baseline (previously for all the spectra baseline correction was done). For IR microscopy, the data were recorded using an imaging detector (MCT array detector) in reflection mode over the $715-4000 \mathrm{~cm}^{-1}$ range, the collection time being $3 \mathrm{~s}$.

SEM analyses were performed on a HITACHI S2600N electron microscope with EDAX, on samples covered with silver layer.

The drug released was evaluated by ultraviolet-visible spectrophotometry based on the peak from $270 \mathrm{~nm}$ using a Thermo Evolution 300 spectrophotometer, in quartz cuvettes of $10 \mathrm{~mm}$ with a scan speed of $240 \mathrm{~nm} / \mathrm{min}$ and data interval of $1 \mathrm{~nm}$ while the bandwidth was set at $1 \mathrm{~nm}$. For this purpose, $1 \mathrm{~g}$ of sample was immersed in $500 \mathrm{~mL}$ phosphate buffer, $0.1 \mathrm{M}$ (7.4 pH), maintained at $37^{\circ} \mathrm{C}$. At fixed time intervals, sample of $5 \mathrm{~mL}$ was extracted and replaced with the same volume of fresh, preheated acceptor phase. All samples were measured in triplicate. 

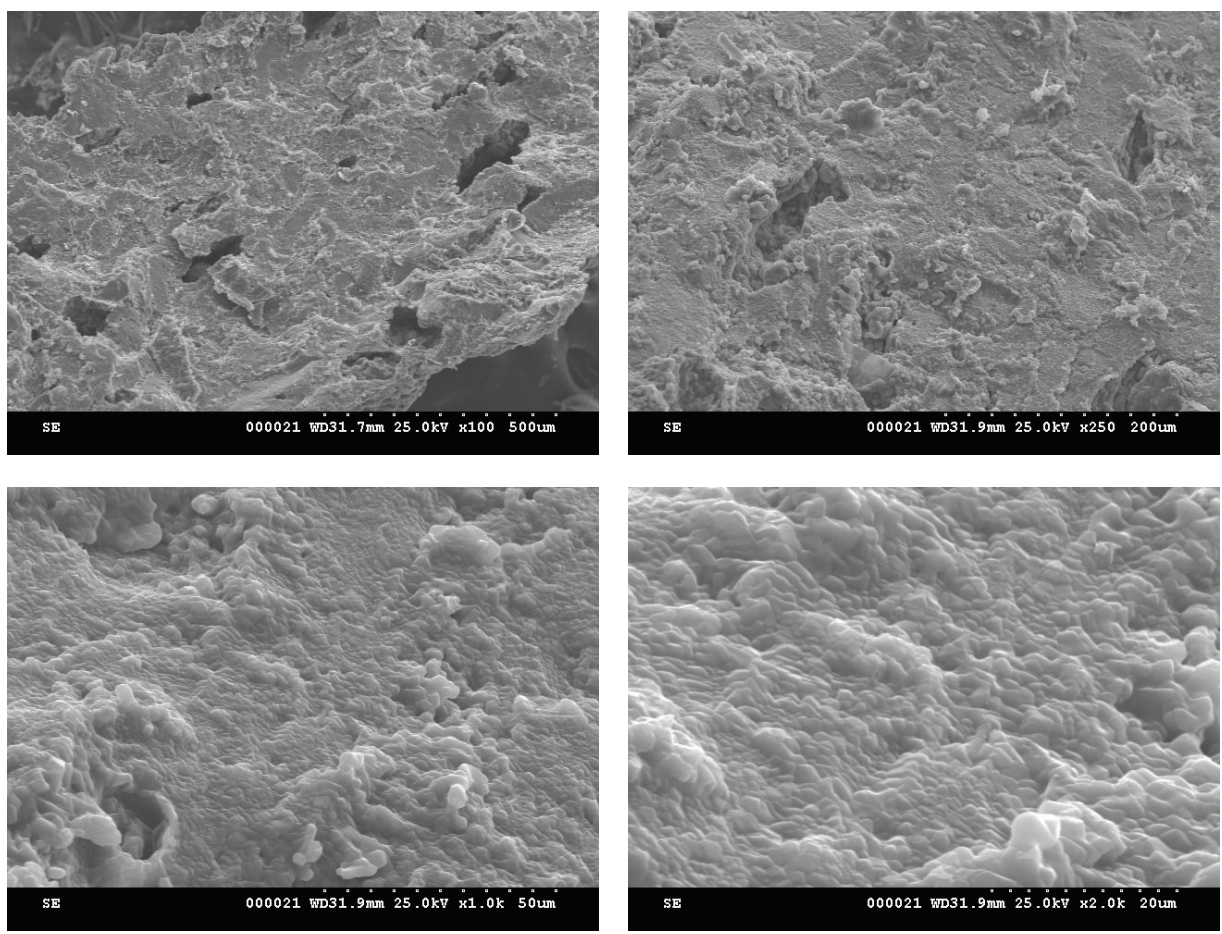

Figure 2: Representative SEM images of COLL-CMC/HA-tetracycline.

\section{Results and Discussion}

Scanning electron microscopy is a useful tool for characterizing materials, the morphology of the materials being essential both for bone grafts and for drug delivery systems. At low magnification (Figure 2 (right-down side)), spherical and ovaloidal pores can be identified, their size being optimal for bone cell colonization $(20-150 \mu \mathrm{m})$ [20-23].

At higher magnification, micronic and submicronic structures can be identified which, based on our previous study, can be the ends of CMC microfibres [24].

Figure 3 presents the experimental spectra of COLL/HA, COLL-CMC/HA, COLL-CMC/HA-tetracycline, tetracycline hydrochloride, and carboxymethyl cellulose.

FTIR spectrum of the COLL-CMC/HA-tetracycline sample exhibits the main absorption bands of collagen, carboxymethyl cellulose, and hydroxyapatite as well as the bands of tetracycline. The intensities of these bands are strongly influenced by composition and molar absorptivity of each characteristic vibration. The low content of tetracycline $(5 \%$ tetracycline) as well as the moderate intensity of its characteristic peaks makes it difficult to clearly identify these peaks. For this reason the deconvolution of the spectrum over the $1200-1800 \mathrm{~cm}^{-1}$ spectral range is necessary. In this range, the deconvolution procedure permits the identification of over 30 absorption bands, some of them being assigned to the main bands of tetracycline $(1235,1283,1384,1406,1468,1515,1551$, $1585,1616,1645$, and $\left.1660 \mathrm{~cm}^{-1}\right)$. It is also worth mentioning that the relative intensity of these bands is similar to that from tetracycline and consequently proves the presence of the drug. Carboxymethyl cellulose can be also identified both in

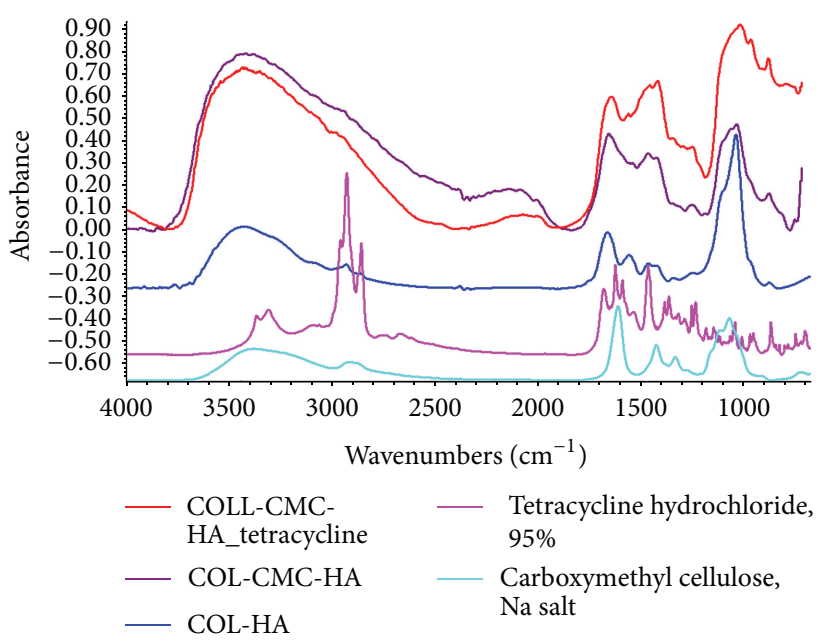

FIGURE 3: FTIR spectra of tetracycline, COLL/HA, and COLL/HAtetracycline.

support material (COLL-CMC/HA) and in the antimicrobial DDS (COLL-CMC/HA-tetracycline). Based on the three experimental spectra, differences can be observed once the number of components increases. For instance, the spectrum of the ternary COLL-CMC/HA composite material differs comparing with the COLL/HA, especially in the region of the absorption bands of CMC $\left(1200-1600 \mathrm{~cm}^{-1}\right)$.

The ternary composite material was also analyzed by FTIR microscopy. The maps were obtained based on the three main independent absorption bands of collagen $\left(1650 \mathrm{~cm}^{-1}\right)$, 


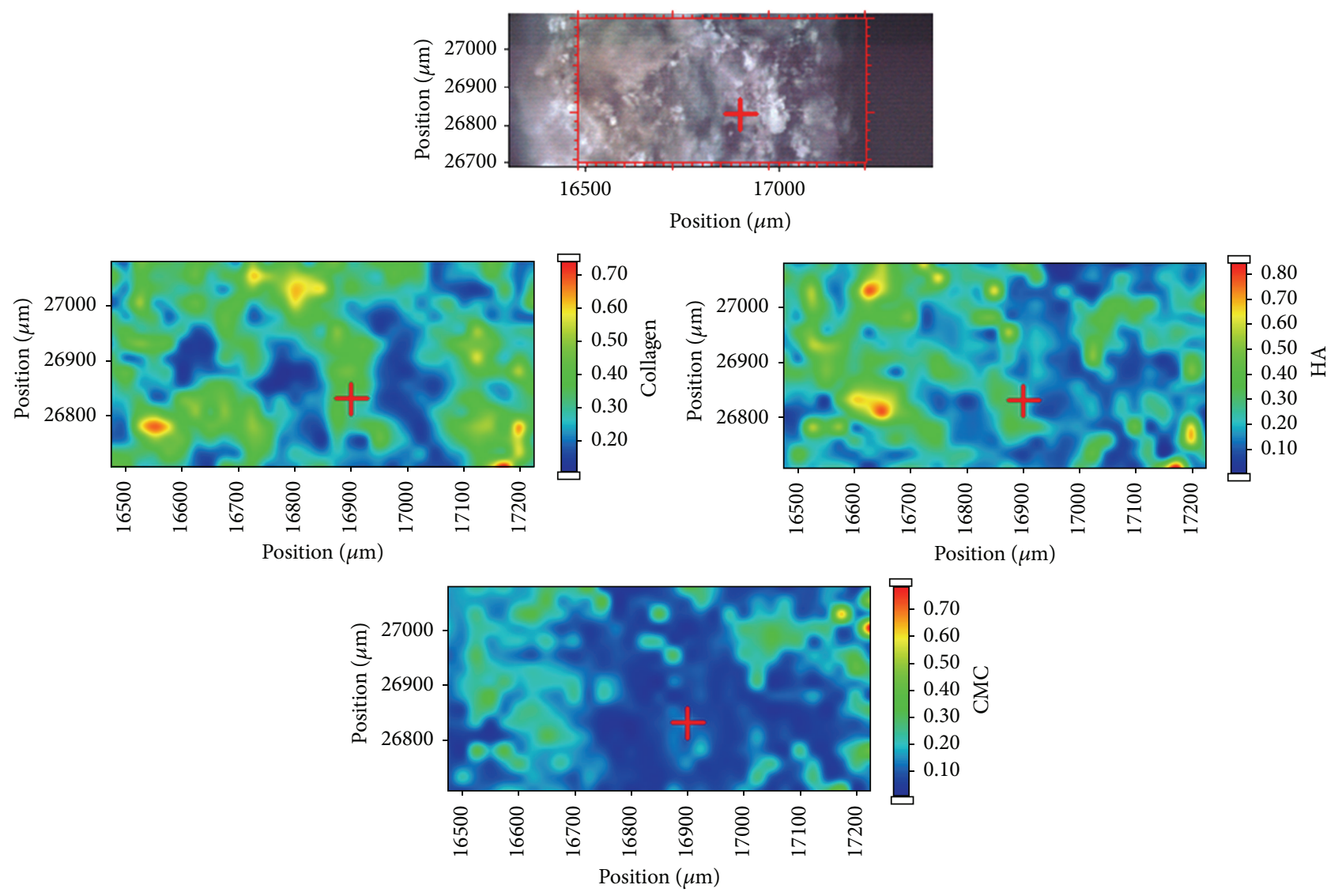

FIGURE 4: FTIR microscopy recorded on COLL-Cell/HA-tetracycline.

hydroxyapatite $\left(1022 \mathrm{~cm}^{-1}\right)$, and cellulose $\left(715 \mathrm{~cm}^{-1}\right)$. The three maps are presented in Figure 4.

The FTIR maps, colored from blue (low intensity) to red (high intensity), reveal the distribution and relative intensity of the monitored bands (components). Based on the correlation with the video image, as well as the relative intensities of the three monitored bands, it can be seen that collagen and hydroxyapatite are intimately associated but also carboxymethylcellulose microfibers are well dispersed into the COLL/HA hybrid composite material, the blue/red distribution being especially a result of the hill/valley topology of the analyzed section.

The release process was monitored by recording the UVVIS spectra of the solutions at different times (Figure 5).

It can be seen that the COLL/HA samples are stable in time, the spectra recorded after three days being identical to that recorded after some minutes of immersion which means that only soluble salts are delivered into the solution. The delivery of tetracycline depends on time. The tetracycline spectra recorded after 1, 3, and 6 days clearly show that the absorbance of both peaks increases. Based on this we can affirm that these systems exhibit prolonged delivery and could be proper candidates as bone grafts for infected bone defects. For quantification, a calibration curve was plotted between $0.1 \mu \mathrm{g} / \mathrm{mL}$ and $10 \mu \mathrm{L}$, the linearity being better than $R^{2}=99.98$. Based on the calibration curve, the correlation

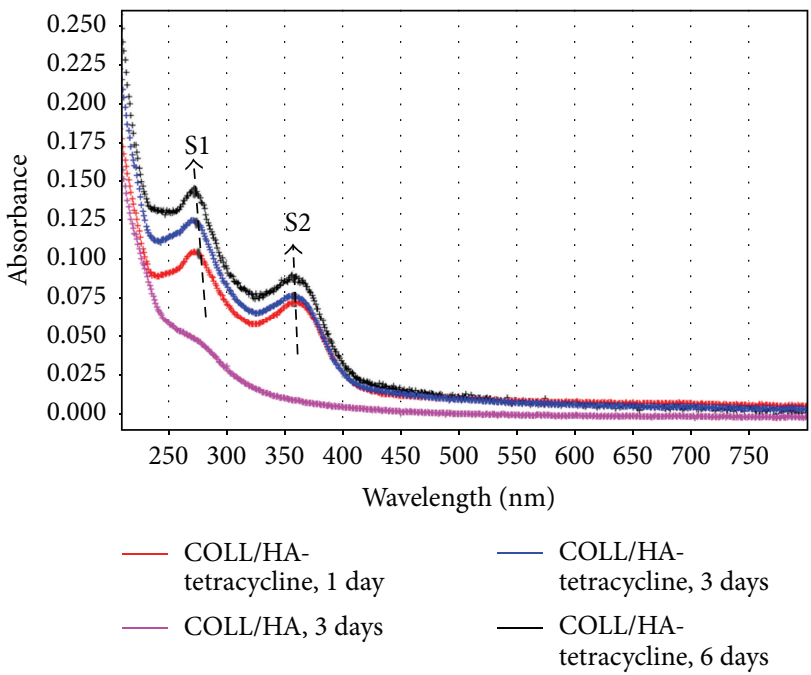

FIGURE 5: UV-VIS spectra of the solution recorded during the delivery process.

between absorbance and concentration is given by the equation $A=0.0896 \cdot c+0.0031$. Comparing with the theoretical concentration, after 6 days, the recovered tetracycline was $79.8 \%$ which is good enough to assure antimicrobial activity during the healing of the wound. In the case of infections, 
over 6 days of delivery could be enough to treat the infections, and, if not, supplementary antibiotic can be administered in classical way.

\section{Conclusions}

New antimicrobial bone grafts with prolonged delivery of tetracycline were obtained by coprecipitation of hydroxyapatite in the presence of the carboxymethylcellulose-collagen gel followed by loading with tetracycline. This material was designed to be used only for infected bone defects. This locoregional delivery of tetracycline is essential to reduce the systemic side effects associated with the use of antibiotics. These materials can be considered with prolonged delivery because even after 6 days the recovery of tetracycline is lower than $80 \%$. This result is consistent with the clinical protocols of treating infections but reduces the amount of tetracycline and reduces the systemic side effects.

\section{Conflict of Interests}

The authors declare no conflict of interests.

\section{Acknowledgments}

This work was supported by Romanian National Authority for Scientific Research, MPNS COST Action MP1301: New Generation Biomimetic and Customized Implants for Bone Engineering and Project no. PIII-C2-PCFI (2015-2016), DENTALOCT.

\section{References}

[1] E. Andronescu, A. Ficai, M. G. Albu et al., "Collagenhydroxyapatite/cisplatin drug delivery systems for locoregional treatment of bone cancer," Technology in Cancer Research and Treatment, vol. 12, no. 4, pp. 275-284, 2013.

[2] A. Ficai, E. Andronescu, D. Ficai, M. Sonmez, M. G. Albu, and G. Voicu, "Mimicking the morphology of long bone," Central European Journal of Chemistry, vol. 10, no. 6, pp. 1949-1953, 2012.

[3] S. V. Dorozhkin, "Calcium orthophosphate-based bioceramics," Materials, vol. 6, no. 9, pp. 3840-3942, 2013.

[4] P. Fratzl and R. Weinkamer, "Nature's hierarchical materials," Progress in Materials Science, vol. 52, no. 8, pp. 1263-1334, 2007.

[5] I.-A. Nedelcu, A. Ficai, D. Ficai, G. Voicu, M. G. Albu, and E. Andronescu, "Hybrid COLL-CMC/HA composite materials for bone tissue regeneration," University Politehnica of Bucharest Scientific Bulletin Series B, vol. 77, no. 1, pp. 3-14, 2014.

[6] A. Ficai, M. G. Albu, M. Sonmez, D. Ficai, and E. Andronescu, "Synthesis of collagen/hydroxyapatite composite materials with oriented microstructure induced by the interaction of the flowing mineralised collagen gel with the support," ChemXpress, vol. 3, pp. 107-110, 2014.

[7] A. Ficai, E. Andronescu, G. Voicu et al., "Self-assembled collagen/hydroxyapatite composite materials," Chemical Engineering Journal, vol. 160, no. 2, pp. 794-800, 2010.

[8] M. Solignac, "New perspectives in antibiotic prophylaxis," Presse Medicale, vol. 27, no. 3, pp. 27-29, 1998.
[9] D. Lebeaux, N. Fernández-Hidalgo, A. Chauhan et al., "Management of infections related to totally implantable venousaccess ports: challenges and perspectives," The Lancet Infectious Diseases, vol. 14, no. 2, pp. 146-159, 2014.

[10] A. Lipp and G. Lusardi, "Systemic antimicrobial prophylaxis for percutaneous endoscopic gastrostomy," The Cochrane Database of Systematic Reviews, no. 11, Article ID CD005571, 2013.

[11] M. Zilberman and J. J. Elsner, "Antibiotic-eluting medical devices for various applications," Journal of Controlled Release, vol. 130, no. 3, pp. 202-215, 2008.

[12] T. Y. Wu, Q. C. Zhang, W. P. Ren et al., "Controlled release of gentamicin from gelatin/genipin reinforced beta-tricalcium phosphate scaffold for the treatment of osteomyelitis," Journal of Materials Chemistry B, vol. 1, no. 26, pp. 3304-3313, 2013.

[13] L. Zhang, Y. Huang, Y. Zhou, T. Buckley, and H. H. Wang, "Antibiotic administration routes significantly influence the levels of antibiotic resistance in gut microbiota," Antimicrobial Agents and Chemotherapy, vol. 57, no. 8, pp. 3659-3666, 2013.

[14] C. García-Rey, J. E. Martín-Herrero, and F. Baquero, "Antibiotic consumption and generation of resistance in Streptococcus pneumoniae: the paradoxical impact of quinolones in a complex selective landscape," Clinical Microbiology and Infection, vol. 12, no. 3, pp. 55-66, 2006.

[15] A. Rodríguez-Rojas, J. Rodríguez-Beltrán, A. Couce, and J. Blázquez, "Antibiotics and antibiotic resistance: a bitter fight against evolution," International Journal of Medical Microbiology, vol. 303, no. 6-7, pp. 293-297, 2013.

[16] A. Dashti, D. Ready, V. Salih et al., "In vitro antibacterial efficacy of tetracycline hydrochloride adsorbed onto Bio-Oss bone graft," Journal of Biomedical Materials Research Part B: Applied Biomaterials, vol. 93, no. 2, pp. 394-400, 2010.

[17] S. Praveen and M. Rohaizak, "Local antibiotics are equivalent to intravenous antibiotics in the prevention of superficial wound infection in inguinal hernioplasty," Asian Journal of Surgery, vol. 32, no. 1, pp. 59-63, 2009.

[18] M. G. Albu, Collagen Gels and Matrices for Biomedical Applications, Lambert Academic Publishing, Saarbrücken, Germany, 2011.

[19] E. Andronescu, M. Ficai, G. Voicu, D. Ficai, M. Maganu, and A. Ficai, "Synthesis and characterization of collagen/hydroxyapatite: magnetite composite material for bone cancer treatment," Journal of Materials Science: Materials in Medicine, vol. 21, no. 7, pp. 2237-2242, 2010.

[20] A. Ficai, E. Andronescu, G. Voicu, and D. Ficai, "Advances in collagen/hydroxyapatite composite materials," in Advances in Composite Materials for Medicine and Nanotechnology, B. Attaf, Ed., chapter 1, InTech, Rijeka, Croatia, 2011.

[21] B.-S. Chang, C.-K. Lee, K.-S. Hong et al., "Osteoconduction at porous hydroxyapatite with various pore configurations," Biomaterials, vol. 21, no. 12, pp. 1291-1298, 2000.

[22] H. Develioglu, E. Koptagel, R. Gedik, and L. Dupoirieux, "The effect of a biphasic ceramic on calvarial bone regeneration in rats," Journal of Oral Implantology, vol. 31, no. 6, pp. 309-312, 2005.

[23] O. Gauthier, J.-M. Bouler, E. Aguado, P. Pilet, and G. Daculsi, "Macroporous biphasic calcium phosphate ceramics: influence of macropore diameter and macroporosity percentage on bone ingrowth," Biomaterials, vol. 19, no. 1-3, pp. 133-139, 1998.

[24] D. E. Fetterolf and R. J. Snyder, "Scientific and clinical support for the use of dehydrated amniotic membrane in wound management," Wounds, vol. 24, no. 10, pp. 299-307, 2012. 

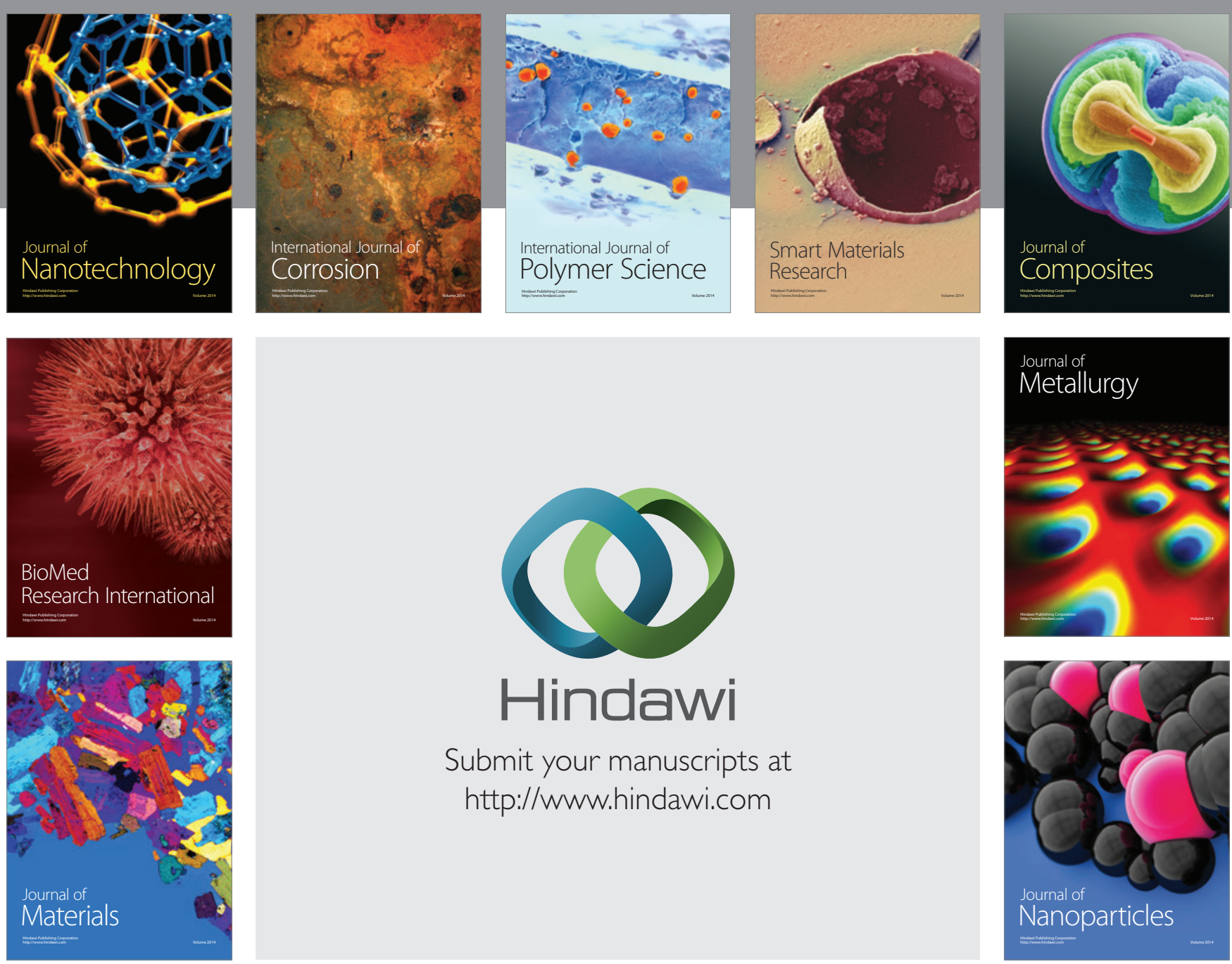

Submit your manuscripts at http://www.hindawi.com
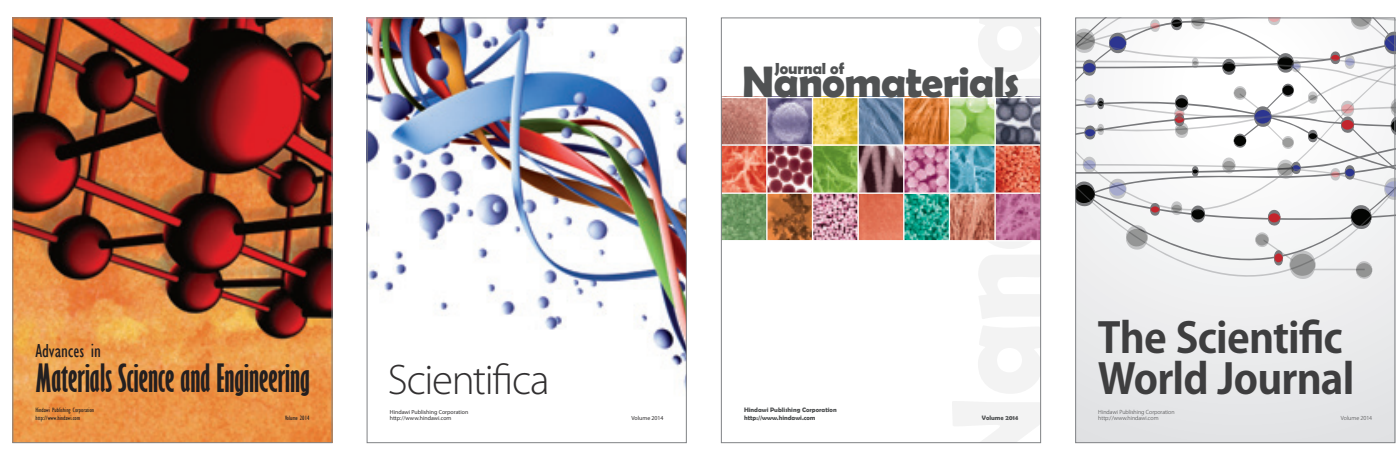

\section{The Scientific World Journal}
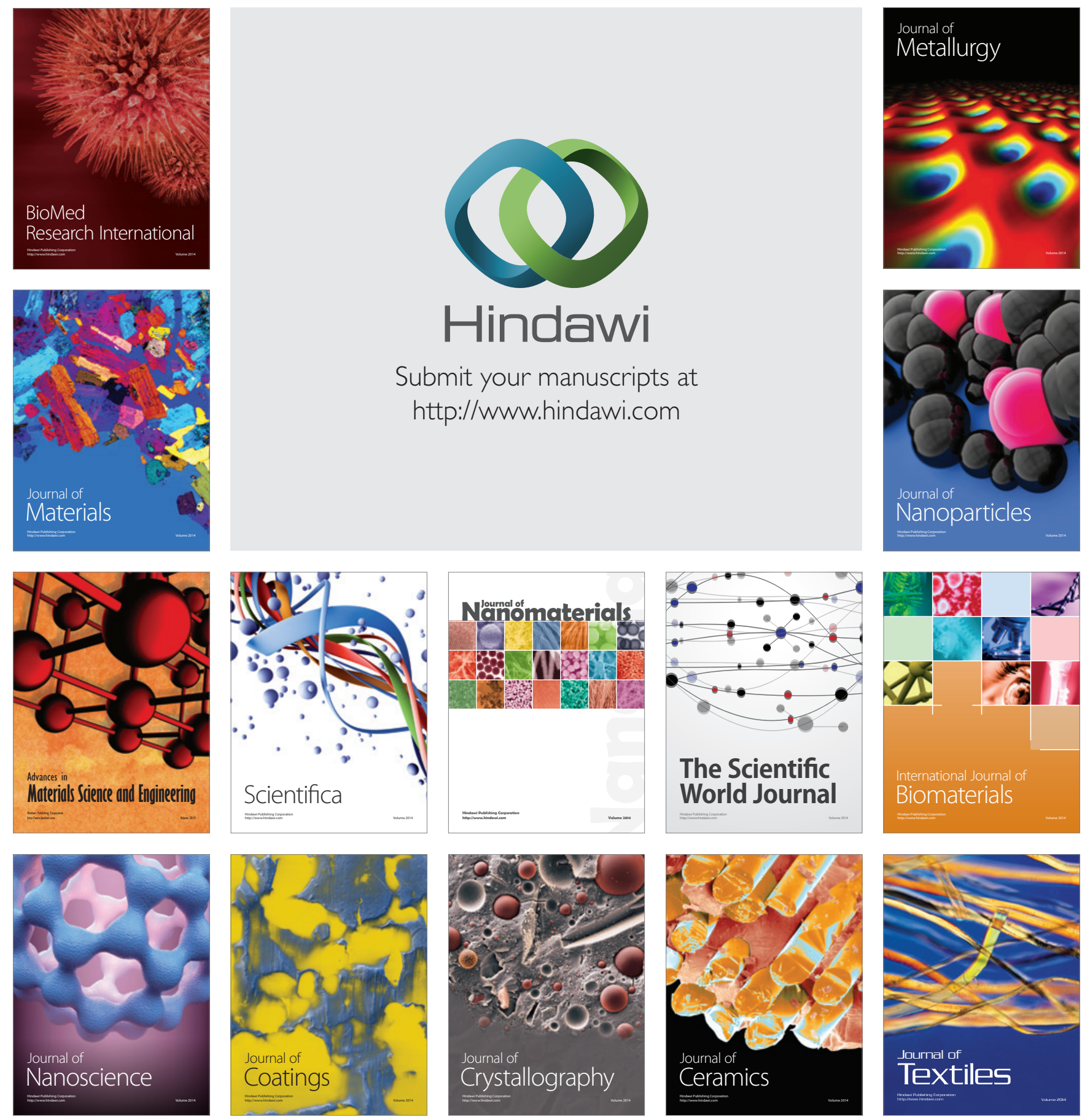